\title{
24 hour ambulatory oesophageal motility monitoring: How should motility data be analysed?
}

\author{
J E RICHTER AND D O CASTELL
}

From the Gastroenterology Section, Bowman Gray School of Medicine of Wake Forest University, WinstonSalen, North Carolina, USA

SUMMARY Ambulatory oesophageal motility/pH monitoring permits accurate detection of oesophageal events during spontaneous chest pain episodes. Opinions differ, however, about the methods to review the extensive motility data and the definition of abnormal motility changes. We studied 30 patients (18 women, age 46 years) with suspected oesophageal chest pain using a portable recording system attached to a $4.5 \mathrm{~mm}$ catheter with pressure transducers 3 and $8 \mathrm{~cm}$ and pH probe $5 \mathrm{~cm}$ above the lower oesophageal sphincter (LOS). An event marker was triggered by the patient for chest pain. In the patient's diary, pain was recorded on a scale of increasing severity 1-10. Two methods of analysis were used to assess 24 hour motility data. The 24 hour technique sampled five minute asymptomatic baselines throughout the study to define the patient's normal range of oesophageal motility. The second technique used only the 10 minute period immediately before each chest pain episode as the asymptomatic baseline. Chest pain episodes were defined as abnormal if associated with $\mathrm{pH}<4$ or motility changes not present during the asymptomatic baseline analysis: 135 chest pain episodes were recorded. The method of motility analysis significantly $(\mathbf{p}<0.01)$ changed the number of chest pain episodès associated with abnormal motility: 24 hour technique - 14 episodes $(10 \%)$ versus a $2 \cdot 5$-fold increase with the 10 minute baseline technique -33 episodes $(24 \%)$. Acid related pain episodes were similar in both groups $13 \%$. The majority of chest pain episodes had no association with abnormal motility or acid reflux. Increasing chest pain severity was inversely correlated with the presence of abnormal oesophageal events. We conclude that limited analysis of 24 hour motility data may over diagnose motility related chest pain events and lead to inappropriate medical or surgical therapy.

Twenty four hour oesophageal ambulatory motility and $\mathrm{pH}$ monitoring is a new research tool for the investigation of non-cardiac chest pain. This technique offers several advantages over conventionally available oesophageal tests: (1) it permits evaluation of multiple, spontaneous chest pain episodes occurring over a prolonged period of time, (2) patients can be studied as outpatients in their home or work environments, and (3) this system allows a direct correlation between the patient's complaints of chest pain and oesophageal motility activity and acid reflux.

Address for correspondence: Joel E Richter, MD, Gastroenterology Section, Bowman Gray School of Medicine, 300 S Hawthorne Road, Winston-Salem, North Carolina, 27104 USA.

Accepted for publication 4 January 1989.
Several laboratories have been developing ambulatory motility systems, ${ }^{1-4}$ but only two have reported clinical studies in patients with non-cardiac chest pain. Janssens $e t$ al found that 24 hour recordings showed the oesophagus to be the likely cause of symptoms in 21 of 60 patients (35\%) with 'severe', recurrent angina like chest pain. ${ }^{\prime}$ More recently, we have reported our experience in 22 patients with a total of 92 spontaneous chest pain episodes. ${ }^{2}$ Eleven events $(12 \%)$ occurred in association with abnormal oesophageal motor activity while 18 events $(20 \%)$ were associated with acid reflux, and four events (4\%) had both abnormal activities. To our surprise, the majority of chest pain episodes, 59 events $(64 \%)$ did not have any association with either abnormal oesophageal motility or $\mathrm{pH}$. 
Unlike oesophageal $\mathrm{pH}$ studies, there is no concensus about how to define abnormal oesophageal pressures as the cause of chest pain during ambulatory motility studies. The Belgium group has suggested that: 'a pain episode correlated in time with severe motility abnormalities that were not present in other parts of the recording... makes the chest pain likely of oesophageal origin'.' This concept allows for a subjective, but not very objective, criterion to assess 'abnormality' on recordings of this kind. Therefore, the primary goal of this study was to begin to define how much of the asymptomatic motility data must be analysed to fulfil a criterion similar to that suggested by Janssens et al. Do we need to meticulously review the entire 24 hour motility tracing or is it sufficient to simply analyse the data occurring immediately before a chest pain episode? A secondary goal was to determine whether or not increasing pain severity identifies the presence of abnormal oesophageal activity. In our experience, there is no indication that more severe pain, as reported by patients, predicts the presence of an oesophageal origin of pain. Others have found a high association of oesophageal events when their patients experienced severe, cramping, retrosternal pain.'

\section{Methods}

PATIENTS

Thirty patients (18 women) with the mean age of 46 years (range 25-68) were studied. All patients had suffered from severe, recurrent substernal chest pain for an average of two years. At the time of this study, 25 patients were having daily chest pain and the remaining five patients reported pain every other day. Extensive cardiac, pulmonary, musculoskeletal and gastrointestinal evaluations were performed on these patients before entry to this study. Coronary arteriography showed normal coronary arteries in 10 patients and non-obstructive disease (no greater than $25 \%$ coronary artery narrowing) in 15 patients. Five patients only had non-invasive cardiac studies but were considered by their referring cardiologist not to have cardiac chest pain. Pulmonary diseases were excluded by a review of the chest radiographs and normal pulmonary artery pressures when arteriography was performed. A musculoskeletal cause for chest pain was felt not to be present as these patients had no 'trigger points' for the replication of their pain on vigorous palpatation of the anterior and posterior chest wall. All patients had either normal upper gastrointestinal radiographs or panendoscopy. When appropriate, gall stones were excluded by oral cholecystogram or ultrasound. Baseline oesophageal manometry studies were normal in 15 patients. The other patients had abnormal manometry including 10 with the nutcracker oesophagus, two non-specific oesophageal motility disorders, one diffuse oesophageal spasm, one achalasia, and one hypertensive lower oesophageal sphincter." The acid perfusion (Bernstein) test was performed in all patients and reproduced chest pain in 12 individuals. "Sixteen of 29 patients given a placebo controlled intravenous bolus of edrophonium (Tensilon $80 \mu \mathrm{g} / \mathrm{kg}$ ) had a replication of their chest pain.? In an additional seven patients, small volume intraesophageal balloon distention reproduced the patient's pain symptoms.

\section{AMBULATORY SYSTEM}

The ambulatory motility system was similar to that used in our initial study.' Briefly, oesophageal pressures were measured by a Koningsberg P 31 probe (Koningsberg Instruments Company, Pasadena, California) having two transducers mounted $5 \mathrm{~cm}$ apart. The two pressure signals were continuously recorded on a two channel, 24 hour Holter ECG tape recorder (ICR 7200, Instruments for Cardiac Research Inc, East Syracuse, New York). The probe was connected to the recorder through an interface designed by the Medical Physics and Engineering Department of William Beaumont Hospital, Royal Oak, Michigan (US Patent Number $4,503,859)$. At the end of the recording period, the tapes were played back on the ICR Holter Scanner (series 6201) through a custom interface to a two channel chart recorder. Oesophageal $\mathrm{pH}$ was recorded by a DelMar Avionic pH system (DelMar Avionics, Irvine, California). The probe consisted of a $\mathrm{pH}$ glass electrode (Microelectrodes Inc, Londonberry, New Hampshire) with a $1.8 \mathrm{~mm}$ outer diameter and sensitivity of $0.15 \mathrm{pH}$ units. Signals were sampled at the frequency of 10 per minute. A reference electrode was attached to the skin on the anterior chest. Before and after each study, the $\mathrm{pH}$ electrode was calibrated using a neutral buffer and acid buffer of $\mathrm{pH} 4$.

STUDY PROCEDURE

All patients were studied in the outpatient setting after an overnight fast. They were requested to stop any drug therapy known to alter oesophageal pressures or reduce gastric acidity for 24 hours before the study and for the duration of the study. The $\mathrm{pH}$ and motility systems were standardised before each study. The tip of the $\mathrm{pH}$ electrode was positioned between the pressure transducers and secured by silk sutures. The combined probes were placed transnasally and positioned with the pressure transducers at $3 \mathrm{~cm}$ and $8 \mathrm{~cm}$ and the $\mathrm{pH}$ electrode at $5 \mathrm{~cm}$ above the lower oesophageal sphincter as determined by previous manometry. The probes were secured to the face with tape and the individual leads connected to 
the recording units worn on a belt around the waist. Digital clocks on the tape recorder and $\mathrm{pH}$ recorder were synchronised and served as time references for events recorded in the patients diaries. All patients were allowed normal activity and foods of $\mathrm{pH} 5$ or greater. Smoking was allowed and patients were permitted to drink hot and cold liquids as long as the $\mathrm{pH}$ was not less than 5. Patients triggered event markers on the pressure and $\mathrm{pH}$ recordings at the onset of each individual chest pain event. The patients were also asked to keep a diary and record the following events: onset and duration of chest pain episodes, severity of chest pain episodes on a 10 point scale $(1$ - very mild pain to 10 - severe pain requiring medical attention), beginning and ending of meals, and major activities including sleep, exercise, coughing, and bowel movements.

\section{OESOPHAGEAL PRESSURE AND PH DATA \\ ANALYSIS}

During the 24 hour study, each patient served as his or her own control. As summarised in Table 1, two different analysis techniques were used to evaluate the pressure data. The 24 hour analysis technique determined the patient's baseline data during asymptomatic periods by reviewing five minutes samples obtained on the hour as well as five minute random samples of any unusual motility activity observed while scanning all of the patient's asymptomatic motility data. By this method, an average of 84 contraction sequences were measured per person and used to determine the patient's normal asymptomatic motility pattern. The 10 minute baseline analysis technique was similar to that reported by Janssen et al.' In this analysis, the baseline asymptomatic data were limited to the 10 minutes before each individual chest pain episode. Therefore, baseline data for comparison with chest pain episodes could represent anything from no oesophageal activity, as seen when the patient is asleep and awakes with chest pain, to the regular swallowing activity of approximately one contraction per minute. By this method, an average of only seven contraction sequences were measured per person to obtain the asymptomatic baseline before each individual chest pain episode.

Motility parameters measured during the asymptomatic baseline periods were similar for both techniques: mean amplitude and duration, maximum amplitude and duration based on a single wave with the highest contraction amplitude or longest duration, and percent abnormal peristalsis (simultaneous or retrograde). Contraction waves recorded by both transducers were evaluated and wave measurements were done in the same fashion as in conventional manometric studies. For the purpose of
Table 1 Two techniques for the analysis of 24 hour oesophageal motility data

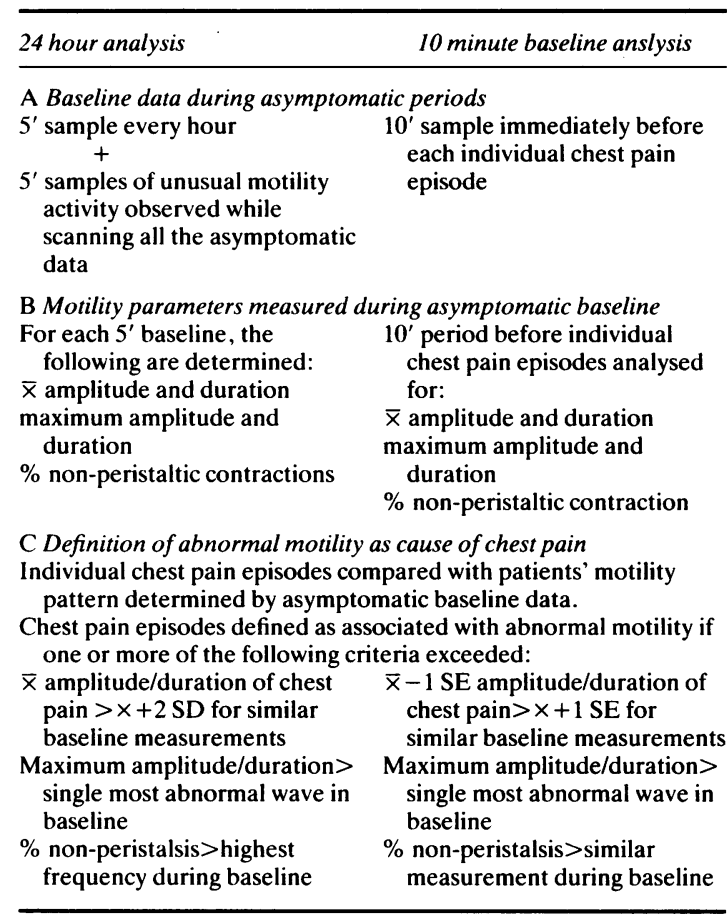

overall data analysis, proximal and distal contraction waves were combined for the determination of mean amplitude and duration.

Individual chest pain episodes were compared with the patient's normal motility pattern as determined by the two analysis techniques for asymptomatic baseline data. All contraction waves during the pain episodes or for five minutes after the onset of pain, if the patient did not identify a specific end point for the pain, were combined and analysed for the same five parameters as the individual asymptomatic baseline periods. A chest pain episode was defined as associated with 'abnormal' motility using the 24 hour analysis technique if one or more of the following criteria were exceeded: (1) mean amplitude or duration greater than mean +2 SD for the same parameters during asymptomatic baseline; (2) maximum amplitude greater than the single contraction wave of highest amplitude observed during the baseline plus the potential artifact arising from respiratory baseline variations in amplitude ( $>35$ $\mathrm{mmHg} ; \times+2 \mathrm{SD}$ for 24 patients); ${ }^{9}$ (3) maximum duration greater than the single contraction wave of longest duration observed during the baseline plus the potential cardiovascular artifact in nonbradycardiac subjects $(>1 \mathrm{sec}) ;{ }^{10}$ and (4) per cent 


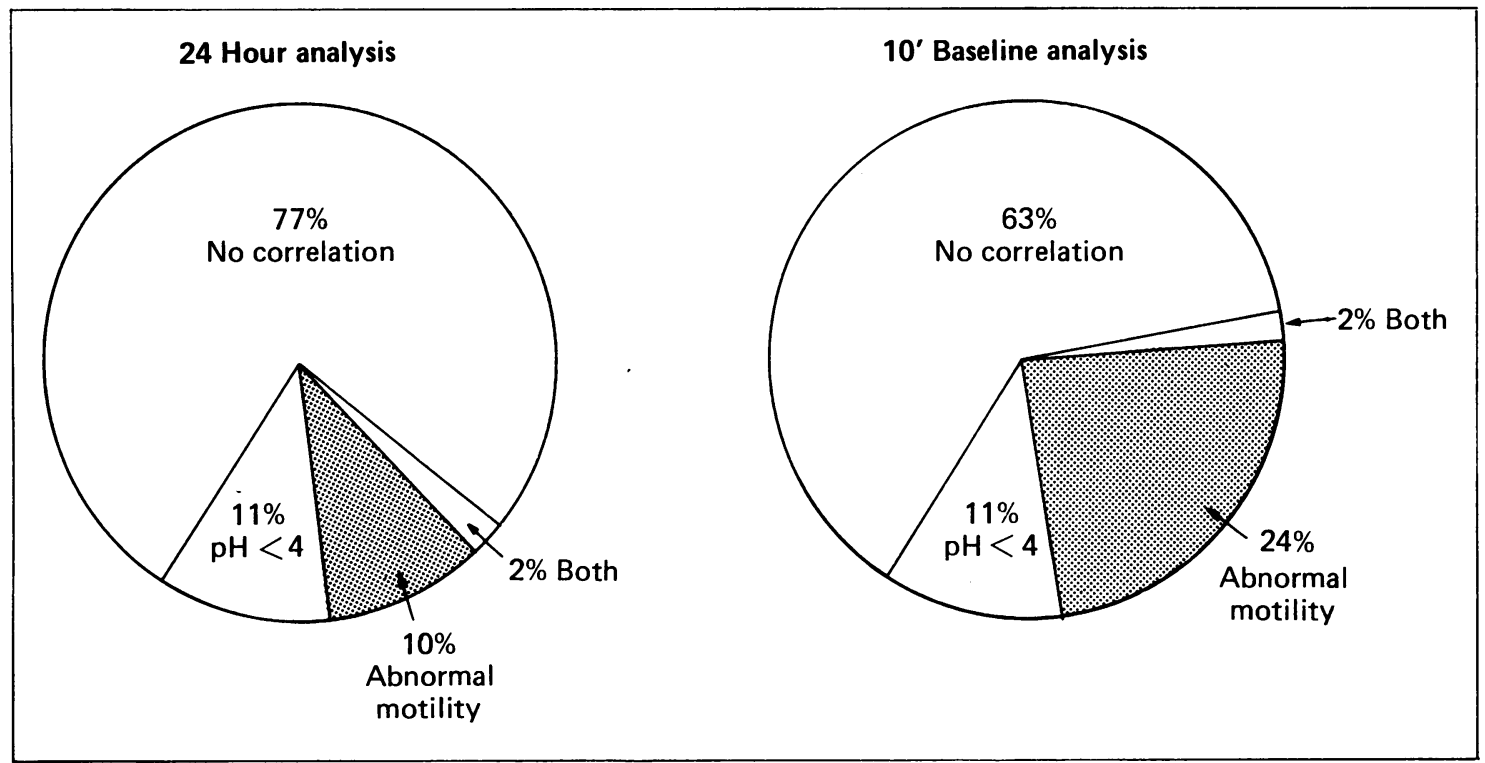

Figure Pie diagram depicting the relationship between the 135 spontaneous chest pain episodes and abnormal oesophageal motility and/or acid reflux $(\mathrm{pH}<4)$ as defined by the 24 hour and 10 minute baseline analysis techniques. The method for analysing motility data significantly $(p<0.01)$ changed the number of chest pain episodes associated with abnormal oesophageal motility (shaded area). Note that regardless of the analysis technique, the majority of chest pain episodes had no association with either abnormal oesophageal motility or acid reflux.

abnormal peristalsis exceeding the highest frequency observed during any patient's asymptomatic baseline. Similar criteria were used for the 10 minute baseline analysis technique except for the determiñnation of abnormal motility based on mean amplitude and duration during the chest pain episode. The small number of contraction waves measured during the baseline period prevented the use of a statistical analysis using standard deviation to describe the normal asymptomatic range of pressures. Therefore, the statistical analysis compared means \pm standard errors - that is, chest pain amplitude $(X-1 \mathrm{SE})>$ asymptomatic baseline amplitude $(x+1 \mathrm{SE})$ for the episode to be defined as associated with 'abnormal motility'. A chest pain episode was defined as $\mathrm{pH}$ associated if it occurred within two minutes of an episode of gastroesophageal reflux ( $\mathrm{pH}$ less than 4). A chest pain episode was considered to be associated with abnormal motility and $\mathrm{pH}$ if the above criteria were met together during the individual chest pain episode. The differences in the two analysis techniques for the 30 patients were compared by $\chi^{2}$ analysis.

The pH parameters initially defined by Johnson and DeMeester ${ }^{10}$ were used to identify the presence of pathological acid reflux. The 24 hour $\mathrm{pH}$ parameters were considered abnormal if the subjects' values exceeded the $95 \%$ confidence level derived from 20 asymptomatic healthy volunteers (eight men, mean age 31 years) previously studied in our laboratory.

In order to evaluate the relationship between chest pain severity and oesophageal events (abnormal pH and/or motility), the chest pain scores derived from the 10 point scale were arbitratily divided into two groups: mild pain - scale 1 to 5 and severe pain - scale 6 to 10 . Chi square analysis was used to compare various relationships between pain groups as determined by the two analysis techniques.

\section{Results}

During the 24 hour ambulatory oesophageal motility and $\mathrm{pH}$ monitoring, all 30 patients experienced replication of their chest pain. These patients noted a total of 135 spontaneous chest pain episodes $(\bar{x} 4 \cdot 5$ episodes per patient, range 1-13). All patients tolerated the procedure well. No procedure related complications were noted.

The association of the 135 spontaneous chest pain episodes with abnormal motility and/or $\mathrm{pH}$ by the two analysis techniques is summarised in the figure. Overall, the 24 hour analysis technique defined 30 chest pain episodes as being associated with abnormal $\mathrm{pH}$ or motility while 51 episodes were identified as abnormal by the 10 minute baseline 
analysis technique. As would be anticipated, the acid related chest pain episodes $(15 ; 11 \%)$ and the combination of acid and motility related episodes $(3 ; 2 \%)$ were the same for both analysis techniques. The method for analysing motility data significantly $(p<0.01)$ changed the number of chest pain episodes associated with abnormal motility. The 24 hour analysis technique identified only 14 chest pain episodes $(10 \%)$ as being associated with severe motility abnormalities that were not present elsewhere during the asymptomatic baseline periods. The shorter 10 minute baseline analysis technique increased by nearly 2.5 fold the number of chest pain episodes associated with abnormal motility. By this technique, 33 individual chest pain episodes $(24 \%)$ were attributed to abnormal motility not observed in the 10 minute period immediately before the chest pain episode. Regardless of the analysis technique, the majority of chest pain episodes had no association with either abnormal oesophageal motility or $\mathrm{pH}$. Overall, the 24 hour analysis technique identified an abnormal oesophageal event as the cause of at least one episode of chest pain in $15 / 30$ patients $(50 \%)$, while the 10 minute baseline analysis technique made a similar identification in $22 / 30$ patients $(73 \%)$.

Patient groups could be subdivided based upon results of 24 hour pH tests. Eleven patients had abnormal acid reflux parameters (five upright, two supine, four both) and 18 patients had normal reflux values. The two analysis techniques did not result in significant differences among the reflux patients: 24 hour analysis - eight abnormal motility (24\%), 11 acid reflux $(27.5 \%)$, two both $(5 \%), 19$ neither $(47.5 \%) v 10$ minute baseline analysis - nine abnormal motility $(22.5 \%) 10$ acid reflux $(25 \%)$, three both $(7 \cdot 5 \%), 18$ neither $(45 \%)$. On the other hand, among the 18 patients with normal 24 hour $\mathrm{pH}$ parameters, the 10 minute baseline analysis identified a significantly greater $(\mathrm{p}<0.01)$ percentage of chest pain episodes associated with abnormal motility: 24 hour analysis - six abnormal motility $(6 \cdot 3 \%)$, four acid reflux $(4 \cdot 2 \%)$, one both $(1 \%), 84$ neither $(88.4 \%) v 10$ minute baseline analysis - 24 abnormal motility $(25 \cdot 3 \%)$, five acid reflux $(5 \cdot 3 \%)$, 66 neither $(69 \cdot 4 \%)$.

Among the five criteria for abnormal motility, maximum duration and amplitude were the most common contraction abnormalities identified by both analysis techniques. Remembering that individual chest pain episodes may have more than one abnormal motility criterion, maximum amplitude or duration was abnormal in $82 \%$ of the motility associated chest pain episodes identified by the 24 hour technique and $86 \%$ of the motility associated chest pain episodes identified by the 10 minute baseline technique. As would be anticipated, varia- tions in pressure criteria for these two contraction abnormalities contributed to most of the differences observed between the two analysis techniques (Table 2).

Only 83 of the 135 chest pain episodes $(61 \%)$ were scored on the 10 point pain scale. By the 24 hour analysis techniques, 13/49 (27\%) mild (score 1-5) and 5/34 (15\%) severe (score 6-10) chest pain episodes were associated with acid reflux and/or abnormal motility. Similarly, 19/49 (39\%) mild and $11 / 34(32 \%)$ severe chest pain episodes were associated with abnormal oesophageal activity when a 10 minute baseline analysis was used. As shown in Table 3 , there was an inverse relationship between patients'

Table 2 Variation in pressure criteria based on analysis used

\begin{tabular}{|c|c|c|c|}
\hline \multirow[b]{2}{*}{ Patient initials } & \multicolumn{3}{|c|}{ Maximum amplitude $(\mathrm{mmHg})$} \\
\hline & $\begin{array}{l}\text { Chest pain } \\
\text { episodes }\end{array}$ & $\begin{array}{l}10^{\prime} \\
\text { baseline }\end{array}$ & $\begin{array}{l}24 \text { hour } \\
\text { baseline }\end{array}$ \\
\hline \multicolumn{4}{|c|}{ Abnormal by both analysis: } \\
\hline \multirow[t]{2}{*}{ SA } & 140 & 30 & 100 \\
\hline & 170 & 15 & 100 \\
\hline \multirow[t]{2}{*}{ DM } & 240 & 180 & 200 \\
\hline & 240 & 150 & 200 \\
\hline $\mathrm{CH}$ & 230 & 160 & 180 \\
\hline \multicolumn{4}{|c|}{ Abnormal by $10^{\prime}$ analysis only: } \\
\hline \multirow[t]{2}{*}{ SS } & 270 & 120 & 360 \\
\hline & 200 & $0^{*}$ & 360 \\
\hline \multirow[t]{2}{*}{ SA } & 100 & 50 & 100 \\
\hline & 70 & $0^{*}$ & 100 \\
\hline \multirow{2}{*}{ MP } & 170 & 100 & 285 \\
\hline & 100 & 0 * & 285 \\
\hline MJ & 200 & 170 & 250 \\
\hline \multirow[t]{2}{*}{ MD } & 160 & 80 & 260 \\
\hline & $\begin{array}{l}\text { Maximum } \\
\text { duration (se }\end{array}$ & & \\
\hline \multicolumn{4}{|c|}{ Abnormal by both analyses: } \\
\hline MS & 22 & 14 & 18 \\
\hline \multirow[t]{2}{*}{ ES } & 10 & $0 *$ & 8 \\
\hline & 14 & 7 & 8 \\
\hline JH & 15 & 8 & 10 \\
\hline \multirow{2}{*}{ BK } & 15 & 9 & 11 \\
\hline & 13 & 10 & 11 \\
\hline JE & 10 & 6 & 8 \\
\hline \multirow[t]{2}{*}{$\mathrm{HH}$} & 18 & 14 & 16 \\
\hline \multirow{2}{*}{\multicolumn{4}{|c|}{ Abnormal by $10^{\prime}$ analysis only: }} \\
\hline & & & \\
\hline \multirow[t]{2}{*}{ FJ } & 12 & 9 & 13 \\
\hline & 9 & $0^{*}$ & 13 \\
\hline JP & 6 & 4 & 11 \\
\hline ES & 5 & $0^{*}$ & 8 \\
\hline BK & 9 & 6 & 11 \\
\hline $\mathrm{JC}$ & 5 & 3 & 9 \\
\hline MB & 7 & 5 & 10 \\
\hline DB & 6 & 5 & 8 \\
\hline $\mathrm{HH}$ & 11 & 8 & 16 \\
\hline
\end{tabular}

*No pressure waves in the 10 minutes before chest pain episode; four of six occurred while patient sleeping. 
Table 3 Relationship between abnormal oesophageal events and chest pain severity

\begin{tabular}{lll}
\hline & $\begin{array}{l}\text { 24 hour analysis* } \\
\% \text { of abnormalloesophageal events }\end{array}$ & $\begin{array}{l}10 \text { minute baseline analysis } \\
\text { Pain scale }\end{array}$ \\
$1-5$ & $72 \%$ & $63 \%$ \\
$6-10$ & $28 \%$ & $37 \%$ \\
& $\mathrm{p}<0.001$ & $\mathrm{p}<0.05$ \\
\hline
\end{tabular}

*Percentage derived from number of abnormal oesophageal events in each pain group over the total number of chest pain episodes caused by acid reflux and/or abnormal motility.

pain scores and the likelihood that abnormal oesophageal activity caused the chest pain. Thus, acid reflux and/or abnormal motility was significantly less likely to occur with the more severe pain episodes. In patients experiencing multiple chest pain episodes, there also was no evidence that they could distinguish chest pain events associated with abnormal motility of $\mathrm{pH}$ from chest pain events observed during normal oesophageal activity.

\section{Discussion}

The purpose of this current study was to address the question: how much motility data must be analysed to confidently identify a chest pain episode as being caused by abnormal oesophageal contractile activity? To this end, one must first agree upon the definition of abnormal oesophageal motility. On the surface, this may seem a simple question. Unlike acid reflux or blood pressure, however, where an abnormal physiological cutoff has been defined, the definition of abnormal motility is less clear. Traditionally, normal ranges for oesophageal contractile parameters (amplitude, duration, velocity, per cent simultaneous, and non-conducted contractions) have been determined by studying a large number of healthy subjects with stationary manometry and standardised swallowing techniques. ${ }^{11-14}$ For a given parameter, a mean value and \pm 2 (or even 3 ) standard deviations would be determined. Any measurement exceeding these normal limits would be defined as abnormal. From a statistical standpoint, this is a valid technique but gives us no assurance that these 'abnormal' contractions represent an aberrant physiological state or disease that causes chest pain. In fact, these patients are usually asymptomatic at the time the abnormal oesophageal contractions are being recorded. It is quite difficult, therefore, to determine whether these 'abnormal' contractions are the cause of the patient's chest pain or merely represent that particular patient's asymptomatic pressure profile. Our recent experience treating non-cardiac chest pain patients with calcium channel blockers would strongly caution against the former conclusion. Nifedipine significantly decreased contraction pressures in patients with the nutcracker oesophagus but these changes did not result in improvement in chest pain. ${ }^{15}$ On the other hand, 24 hour ambulatory oesophageal motility allows the patient to serve as his or her own control. This eliminates the somewhat artificial comparison with asymptomatic control subjects. Furthermore, this techniques permits a more representative assessment of the patients' normal motility pattern during wet and dry swallows as well as the many daily activities (eating, drinking, sleeping) not associated with chest pain. When the patient experiences chest pain, an event marker is triggered and the motility parameters during chest pain can be directly compared with the patients' asymptomatic motility pattern.

Janssens $e$ t al have recently suggested that 'a pain episode correlated in time with severe motility abnormalities that were not present in other parts of the recording ... makes the chest pain likely of esophageal origin'.' Although some may think this too strict a criteria, we believe this relationship needs to be met before we can confidently attribute a patient's chest pain to oesophageal motility abnormalities. Furthermore, we must strive to quantify this large amount of motility data in order to base our evaluation on objective rather than subjective comparisons. The motility parameters analysed in this study are those measurements (mean and maximum amplitude and duration, percentage abnormal peristalsis) which have traditionally been thought to cause oesophageal chest pain. ${ }^{16}$ Although arbitrary and somewhat difficult to calculate, these motility criteria meet the standards suggested by the Belgium group. To date, they are also the only published reference points for the definition of abnormal motility during 24 hour oesophageal pressure monitoring. Further refinement and justification for this data analysis will await studies from other oesophageal laboratories interested in the evaluation of non-cardiac chest pain.

Our study suggests that the amount of motility data reviewed and analysed while the patient is pain free is a critical factor in determining the relationship between abnormal motility and chest pain. Although the 10 minute baseline analysis is rapid and easy to calculate, it really undermines the purpose of 24 hour oesophageal motility monitoring. By this technique, 33 chest pain episodes were determined to be associated with abnormal oesophageal motility. Review of the entire 24 hour tracing, however, found that the majority ${ }^{17}$ of these episodes would not fulfil our criteria for abnormality. That is, these motility changes were no different than other period of pressure activity recorded when the patients were 
asymptomatic. Therefore, more limited pressure analysis may markedly overestimate motility related chest pain events and possibly lead to inappropriate medical or surgical therapy.

Van Trappen and colleagues (personal communication) have suggested that limiting the baseline analysis to the 10 minutes before chest pain more appropriately takes into account possible changes in oesophageal pain thresholds. Studies with somatic pain have shown that a preceeding stimulus may change the receptor in such a way that the next, identical stimulus elicits a quite different response at the same receptor site. ${ }^{17} 18$ These studies were done with heat injury, however, $\left(50^{\circ} \mathrm{C}\right.$ for 100 second duration) sufficient to cause erythema and discomfort for several days. In contrast with somatic pain receptors, these observations have not been noted with visceral pain receptors. In fact, almost all the available data refer to somatic rather than visceral pain thresholds as assessed by electrical or heat stimulation. Using limited baseline pressure data, who is to say whether a 10 minute, 20 minute, or one hour baseline assessment before chest pain is appropriate? Until more data are available about variable visceral pain thresholds, analysis of the total 24 hour pressure data seems more logical and defensible.

In our experience, the majority of patients with non-cardiac chest pain have their symptoms daily or every other day and frequently have multiple chest pain episodes over 24 hours. One might hope that pain severity would predict the presence of abnormal oesophageal events and thereby eliminate the need to analyse minor pain episodes. The present study indicates, however, that the patient's subjective perception of pain severity is a poor predictor of the presence of abnormal oesophageal events. In fact, abnormal oesophageal events were significantly less frequent during the more severe chest pain episodes. These observations are consistent with the concept that pain is a complex process mediated by factors such as personality traits, mood status, individual cultural values, and environmental events. ${ }^{19}$ Pain assessment may be particularly difficult in noncardiac chest pain patients because they have a high prevalence of underlying psychiatric disorders ${ }^{2021}$ and are generally sensitive to a variety of oesophageal stimuli, many of which normally do not cause chest pain in healthy subjects. ${ }^{2123}$

The development of 24 hour oesophageal $\mathrm{pH}$ monitoring has markedly improved our ability to associate a variety of symptoms with abnormal gastro-oesophageal reflux. It is hoped that 24 hour oesophageal motility monitoring will add the same refinement to the association of non-cardiac chest pain with oesophageal motility disorders. Current ambulatory motility systems allow us to accurately record oesophageal pressures in the patient's home and work environment. In the future, computerised analysis will allow the rapid interpretation of this large data base as has occurred with oesophageal $\mathrm{pH}$ monitoring. The definition of abnormality, however, and clarification of preferred analysis techniques must be developed by the human investigators. This study is an attempt to begin to address these important questions.

The ambulatory motility system used in this study was developed by Dale Petty, CCE in collaboration with Luis Maas, MD and David Penner, MD of the William Beaumont Hospital, Royal Oak, Michigan, USA. The authors also thank Christine Dalton, PAC for technical assistance and Kathy Myers for secretarial preparation of this manuscript. This work was presented at the XI International Symposium on Gastrointestinal Motility in Oxford, England on 9 September 1987 and was published in abstract form in Dig Dis Sci 1987; 32: 924.

\section{References}

1 Janssens J, Vantrappen G, Ghillebert S. 24 hour recording of esophageal pressure and $\mathrm{pH}$ in patients with non-cardiac chest pain. Gastroenterology 1986; 90: 1978-84.

2 Peters L, Maas LC, Petty D, et al. Spontaneous noncardiac chest pain: Evaluation by 24 hour ambulatory motility and $\mathrm{pH}$ monitoring. Gastroenterology 1988; 94: 878-86.

3 Maas LC, Gordon RK, Penner D, et al. 24 hour ambulatory manometry in diagnosis of esophageal motor disorders causing chest pain. South Med J 1985; 78: 810-3.

4 Smout AJPM, Breedijk M, Van der Zouw C, Akkermans L. A new system for continuous 24 hour esophageal pressure and $\mathrm{pH}$ recording with automated analysis. Gastroenterology 1986; 90: 1641.

5 Katz PO, Dalton CB, Richter JE, Wu WC, Castell DO. Esophageal testing of patients with noncardiac chest pain or dysphagia. Ann Intern Med 1987; 106: 593-7.

6 Bernstein LM, Baker LA. A clinical test for esophagitis. Gastroenterology 1958; 34: 760-81.

7 Richter JE, Hackshaw BT, Wu WC, Castell DO. Edrophonium: a useful provocative test for esophageal chest pain. Ann Intern Med 1985; 103: 14-21.

8 Barish CF, Castell DO, Richter JE. Graded esophageal balloon distention: a new provocative test for noncardiac chest pain. Dig Dis Sci 1986; 31: 1292-8.

9 Clouse RE, Staiano A, Landau DW, Schlacter JL. Manometric findings during spontaneous chest pain with presumed esophageal 'spasms'. Gastroenterology 1983; 85: $395-402$.

10 Johnson LF, DeMeester TR. Twenty-four hour $\mathrm{pH}$ 
monitoring of the distal esophagus: a quantitative measure of gastroesophageal reflux. Am J Gastroenterol 1974; 62: 325-32.

11 Dodds WJ, Hogan WJ, Reid DP, Stewart EI, Arndorfer RC. A comparison between primary esophageal peristalsis following wet and dry swallows. J Appl Physiol 1973; 35: 851-7.

12 Hollis JB, Castell DO. Effects of dry swallows and wet swallows of different volumes on esophageal peristalsis. 1975; 38: 1161-4.

13 Clouse RE, Staiano A. Contraction abnormalities of the esophageal body in patients referred for manometry: a new approach to manometric classification. Dig Dis $\mathrm{Sci}$ 1983; 28: 784-91.

14 Richter JE, Wu WC, Johns DN, et al. Esophageal manometry in 95 healthy adult volunteers: variability of pressures with age and frequency of 'abnormal' contractions. Dig Dis Sci 1987; 32: 583-92.

15 Richter JE, Dalton CB, Bradley LA, Castell DO. Oral nifedipine in the treatment of non-cardiac chest pain in patients with the nutcraker esophagus. Gastroenterology 1987; 93: 21-8.

16 Fichter JE, Castell DO. Esophageal disease as a cause of non-cardiac chest pain. In: Stollerman GH, Harrington
WJ, LaMont JJ, Lconard JJ, Sipcrstein MD, eds. Advances in internal medicine. Vol 33. Chicago: Year Book Medical Publishers, 1988: 311-35.

17 Fitzgerald M, Lynn B. The sensitization of high threshold mechanoreceptors with myelinated axons by repeated heating. J Physiol 1977; 365: 549-63.

18 Campbell JN, Meyer RA, Lamotte RH. Sensitization of myelinated nociceptive afferents that innervate monkey hand. J Neurophysiol 1979; 42: 1669-79.

19 Melzack R. Wall PD. The challenge of pain. New York: Basic Books, 1983.

20 Clouse RE, Lustman JJ. Psychiatric illness and contraction abnormalities of the esophagus. $N$ Engl J Med 1983; 309: $1337-42$.

21 Richter JE, Obrecht WF, Bradley LA, Young LD, Anderson KO. Psychological comparison of patients with nutcracker esophagus and irritable bowel syndrome. Dig Dis Sci 1986; 31: 131-8.

22 Richter JE, Barish CF, Castell DO. Abnormal sensory perception in patients with esophageal chest pain. Gastroenterology 1986; 91: 845-52.

23 Vantrappen G, Janssens J, Ghillebert G. The irritable oesophagus - a frequent cause of angina-like chest pain. Lancet 1987; i: 1232-4. 